\title{
The treatment and prevention of acute ischemic stroke
}

$\mathrm{P}$ rotecting the brain from the consequences of vascular obstruction is obviously very important. However, how to manage this is a very complex task. Three recent studies have provided evidence that should not be ignored or misinterpreted by physicians. ${ }^{1-3}$

The National Institute of Neurological Disorders and Stroke rt-PA Stroke Study Group' shows the results of a randomized, collaborative placebo-controlled trial. Six hundred and twenty-four patients were studied. The treatment was started before 90' of the start of the stroke or before 180'. Patients received recombinant rt-PA (alteplase) $0.9 \mathrm{mg} / \mathrm{kg}$ or placebo. A careful neurological evaluation was done at 24 hours and 3 months after the stroke.

There were no significant differences between the groups at 24 hours after the stroke. The evaluation done at 3 months showed that there were more patients in the treated group with minimal to no disability than in the placebo group ( $50 \%$ vs. $38 \%$ p $<0.03)$.

In other words, 1 out of 8 treated patients was spared a neurological disability. On the other hand, symptomatic intracerebral haemorrhage was ten times more frequent in the group that received rt-PA $(6.4 \%)$ than in the control $(0.6 \%) \mathrm{p}<0.001$.

Another study, Low-molecular weight Heparin (LMWH) for the Treatment of Acute Ischemic Stroke, ${ }^{2}$ compared subcutaneous injections of low-molecular heparin, 4100 antifactor Xa IU of nadroparin calcium in $0.4 \mathrm{ml}$ of solution every 12 hours (higher dose), LMWH

* $M D, P h D, M S c$

Chairman, Department of Internal Medicine, Escola

Paulista de Medicina, Editor, São Paulo Medical Journal. alternated with placebo (lower dose) and placebo injections every 12 hours, for 10 days. The evaluation at 6 months showed that the treated group had a lower incidence rate of poor outcomes, death or dependency in daily activities, 45 vs. $65 \%$. In other words, it was necessary to treat 5 patients to benefit one. Evaluations at 10 days did not show differences in death rates or haemorrhage transformation of the cerebral infarction.

The Multicentre Acute Stroke Trial-Italy (MAST-I) Group compared the effectiveness of streptokinase, aspirin and a combination of both for the treatment of ischemic stroke. Six hundred and twenty-two patients were randomized to receive 1 hour infusions of $1.5 \mathrm{mU}$ of streptokinase alone, the same dose of streptokinase plus $300 \mathrm{mg}$ of buffered aspirin, or $300 \mathrm{mg}$ of aspirin alone for 10 days, or nothing. The case fatality rate was higher in the groups treated with streptokinase alone, or streptokinase plus aspirin, than in the group that received only aspirin or nothing.

Inspite of all this information, more studies are necessary to establish safe and effective guidelines for the treatment of schemic stroke. It appears that aspirin and low-molecular weight heparin are safe, but new studies are necessary to compare aspirin with LMWH and with rt-PA.

This will require collaborative studies, and then good randomized trials must be analyzed as a whole using a systematic review. Interested readers may refer to a very interesting commentary by Longstreth in Evidence Based Medicine. ${ }^{4}$ Although the best treatment for ischemic stroke it not yet well established, its prevention is more clear. Treatment of arterial hypertension, particularly in the elderly, ${ }^{5}$ and the long standing use of low-dose aspirin in high-risk patients brings benefits. ${ }^{6}$ In regards to ischemic stroke, as usual, prevention is better than treatment. 


\section{REFERENCES}

1. The National Institute of Neurological Disorders and Stroke rt-PA Stroke Study Group. Tissue Plasminogen activator for acute ischemic stroke. N Engl J Med 1995;333:1581-7.

2. Kay R, Wong KS, Yu YL, et al. Low-molecular-weight heparin for the treatment of acute ischemic stroke. N Engl J Med 1995;333:1588-93.

3. Multicentre Acute Stroke Trial-Italy (MAST-I) Group. Randomised controlled trial of streptokinase, aspirin, and combination of both in the treatment of acute ischaemic stroke. Lancet 1995;346:1509-14.
4. Longestreth W. Tissue Plasminogen activator improved clinical outcome after acute ischemic stroke - Commentary . Evidence Based Medicine 1996; 4: 106-7.

5. Pearce KA, Furberg CD, Rushing J. Does antihypertensive treatment of the elderly prevent cardiovascular events or prolong life? A meta-analysis of hypertension treatment trials. Arch Fam Med1995;4:943-50.

6. Antiplatelet Trialists' Collaboration. Collaborative overview of randomized therapy - I: Prevention of death, myocardial infarction, and stroke by prolonged antiplatelet therapy in various categories of patients. BMJ 1994; 308:81-106. 